doi:10.17659/01.2017.0111

Journal of Case Reports 2017;7(4):408-410

\title{
Affective Psychosis: A Rare Presentation of Tubercular Meningitis in a Tertiary Care Hospital
}

\author{
Preethi V Reddy ${ }^{1}$, Veda Shetageri ${ }^{2}$, Raghavendra Kumar K ${ }^{3}$, Lavanya Anuroop ${ }^{2}$, Ganeshan Gopalakrisnan ${ }^{2}$ \\ Departments of ${ }^{1}$ Psychiatry and ${ }^{3}$ Child and Adolescent Psychiatry, National Institute of Mental Health and Neurosciences, \\ Bangalore; ${ }^{2}$ Department of Psychiatry, MVJ Medical College and Research Hospital, Bangalore, Karnataka, India.
}

\begin{abstract}
Corresponding Author:
Dr. Preethi V Reddy

Email: drpreethi20@gmail.com

This is an Open Access article distributed under the terms of the Creative Commons Attribution License (creativecommons.org/ licenses/by/3.0).
\end{abstract}

Received Accepted

Published

September 25, 2017

November 29, 2017

December 30, 2017

\begin{abstract}
Background: Tuberculosis (TB) is a disease which has been affecting humanity since archaic ages. Although tubercular meningitis (TBM) is the least commonly observed form of extra-pulmonary TB (5-15\%), it is the most severe form in terms of mortality and morbidity. Case Report: A young female, 2 months post-partum, and a diagnosed case of miliary TB on irregular ATT since one and half month presented with mood and psychotic symptoms. There was no significant improvement noted with regular ATT, antipsychotic and mood stabilizer. Further evaluation revealed TBM with tuberculoma on treatment of which psychiatric symptoms subsided. Conclusion: Atypical presentation of TBM is not uncommon in developing nations like India. We report one such case presenting with syndromal psychiatric symptoms and signs. This report is intended to make psychiatrists aware of the unusual clinical presentations of tubercular meningitis.
\end{abstract}

Keywords: Brain, Meningeal Tuberculosis, Tuberculoma, Psychotic Disorders.

\section{Introduction}

Tuberculosis (TB) is a disease which has been affecting humanity since archaic ages. Although tuberculous meningitis (TBM) is the least commonly observed form of extra-pulmonary TB $(5-15 \%)$ but is the most severe form in terms of mortality and morbidity [1]. Tuberculosis of the nervous system can involve the meninges, brain, spinal cord, cranial and peripheral nerves, ears, and eyes [2]. The usual mental disturbances associated are apathy, irritability, and insidious changes in personality as among the chief characteristics of the early stages of the illness [3]. Tuberculosis may manifest with atypical clinical manifestations and delayed diagnosis may give rise to unexpected grave outcomes [4]. Literature review shows several cases of tubercular meningitis presenting with behavioral and cognitive symptoms but cases presenting as full psychiatric syndrome are very few which have been mentioned later in the text and presentation as mood disorder is even sparse. Thus this case would be a rare report of such a classical presentation.

\section{Case Report}

A 25 year old married female, Mrs R presented with one month history of increased speech, being overfriendly, increased activity, anger outbursts, irritability, expansive ideas, decreased sleep and appetite, decreased selfcare, neglected child care and significant weight loss. Patient was in her $3^{\text {rd }}$ month post-partum, delivered a baby boy, 2 months ago and then developed on and off fever, was diagnosed with miliary tuberculosis and started on anti-tubercular treatment (ATT) category I, child was also started on TB prophylaxis. On discontinuation of ATT, her symptoms worsened gradually. No significant past psychiatric or medical history, neuropsychiatric illness in the family, or significant personal history was elicited. Pre-morbidly she was fairly well adjusted. 
Physical examination revealed mild pallor, weighed $31 \mathrm{kgs}$, BMI: 14, systemic examination were within normal limits initially. After about a week she developed severe headache. On CNS examination neck rigidity was present and Kernig's sign was positive.

On mental status examination she was moderately kempt, poorly nourished, co-operative, eye to eye contact intermittently maintained, easy distractability, disinhibited behaviors noted, overfamiliar, increased psychomotor activity, reaction time decreased, flight of ideas present, delusion of grandiosity, euphoric affect, restricted range and reactivity, labile, inappropriate but congruent, ill-sustained concentration, impaired judgement and absent insight. The provisional diagnosis of organic mood disorder was made.

Routine blood, urine and serum electrolytes were within normal limits except raised ESR. cerebrospinal fluid analysis showed protein: 239 mg/dL, sugar: $50 \mathrm{mg} / \mathrm{dL}$, microscopy: lymphocytes and neutrophils in a proteinaceous background suggesting tubercular picture. Chest X-ray showed miliary shadows, CECT brain: multiple tuberculomas in right temporal and parietal regions with significant peri-lesional edema.

Patient was started on anti-tubercular therapy and olanzapine $5 \mathrm{mg}$ twice daily increased to $15 \mathrm{mg}$ over one week. Psychotic symptoms showed some improvement. Tablet lithium was added due to predominant mood symptoms maintained at $600 \mathrm{mg}$, therapeutic level of $0.82 \mathrm{meq} / \mathrm{L}$, with only minimal improvement. After CNS tuberculosis was confirmed intramuscular injection of streptomycin for 2 weeks, tablet prednisolone, syrup glycerol were added along with the previous treatment. After around one week patient started showing significant improvement in her physical and mood symptoms. YMRS score reduced by $50 \%$ (initial score 42 reduced to 20 ). Patient showed overall $70-80 \%$ improvement on discharge. Lithium and olanzapine were gradually

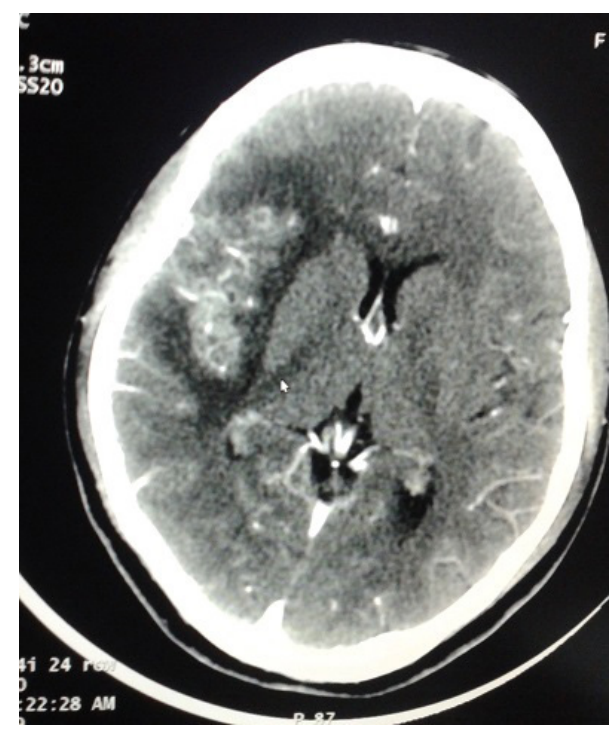

Fig.1: CECT image of the patient, coronal section showing multiple tuberculomas in the right temporo-parietal region with peri-lesional edema.

tapered and stopped over a period of six months and patient is maintaining recovery.

\section{Discussion}

Our literature review showed few case reports of CNS tuberculosis presenting as psychiatric disorders. One of the cases is of anterior callosal and frontal tuberculoma presenting with chronic psychosis and recent onset catatonia [5]. Another case where in mood disturbances in the form of depressive symptoms, cognitive difficulties and hyponatremia were presenting features of tubercular meningitis [6]. A case series about unusual presentations of tubercular meningitis had one of the cases presenting as paranoid psychosis [7]. Another case report from a developing country reported inability to speak, altered behavior which was diagnosed as TBM at a later stage and patient succumbed to his illness [8]. Ziehl-Neelsen staining lacks sensitivity and culture results are often insufficiently timely to aid clinical judgement [9]. Techniques like polymerase chain reaction (PCR) in cerebrospinal fluid and an enzyme linked immunosorbent assay (ELISA) for cerebrospinal fluid antibodies is highly sensitive and gives rapid results [10]. 
The above case would have been considered as a primary mood disorder if not for history of fever, weight loss and being diagnosed as miliary TB, which led to a suspicion of CNS tuberculosis and detailed investigations. What further confirmed the diagnosis is that though psychotic symptoms showed some improvement with olanzapine significant improvement in manic symptoms was seen only after treatment for TB meningitis was initiated. There are few case reports of TBM or CNS tuberculosis presenting as psychosis but manic symptoms are very rare as per our literature search. Thus we present this unique case to sensitize both psychiatrists and physicians about this rare presentation of tubercular meningitis (CNS tuberculosis).

\section{Conclusion}

Psychiatric disorders can be the presenting manifestation of several medical and neurological disorders. In view of severity of the behavioral problems, physical complaints like fever, weight loss, headache might be missed by family members. A high index of suspicion is needed for CNS tuberculosis for patients presenting with physical and psychiatric symptoms. Early diagnosis and treatment definitely reduces morbidity and mortality.

Contributors: PVR: manuscript writing, patient management; VS, LA, GG: Critical inputs into the manuscript, patient management; RKK: manuscript editing and literature review.
PVR will act as guarantor. All authors approved the final version of this manuscript.

Funding: None; Competing interests: None stated.

\section{References}

1. Bozluolcay M, Pelin Z. Tuberculosis of the central nervous system in Turkey: a re-trospective study of 90 adult patients. J of Neurol Sciences. 2003;20:120-126.

2. Saleh M, Saeedi AA, Pooran A. Brain tuberculomas: A case report. Jundishapur J Microbiol. 2014;7:e11252.

3. Williams M, Smith H. Mental disturbances in tuberculous meningitis. J Neurol. Neu-rosurg. Psychiat. 1954;17:173.

4. Togha M, Sahraian MA, Hosseini SJ, Haddadi A. Brain tuberculoma: Report of a case presented with prolonged nonspecific symptoms and multiple brain tuberculoma. Ir J Neurol. 2012;11:34-35.

5. Das S, Gupta R, Dhyani M. Callosofrontal tuberculoma presenting with symptoms of psychosis and catatonia. Indian Journal of Psychiatry. 2015;57:104-105.

6. Benson SM, Narasimhamurthy R. Severe hyponatremia and MRI point to diagnosis of tuberculous meningitis in the Southwest USA. BMJ Case Rep. 2013.

7. Daif A, Obeid T, Yaqub B, Abdul Jabbar M. Unusual presentation of tuberculous me-ningitis. Clin Neurol Neurosurg. 1992;94:1-5.

8. Komolafe MA, Sunmonu TA, Esan OA. Tuberculous meningitis presenting with un-usual clinical features in Nigerians: Two case reports. Bio Med Central. 2008.

9. Thwaites G, Chau TH, Mai NT, Drobniewski F, McAdam K, Farrar J. Tuberculous meningitis- neurological aspects of tropical disease. J Neurol Neurosurg Psychiatry. 2000;68:289-299.

10. Shankar P, Manjunath N, Mohan KK. Rapid diagnosis of tuberculous meningitis by polymerase chain reaction. Lancet. 1991;337:5-7. 